\section{AUTISM SPECTRUM (ASD) AND ATTENTION DEFICIT HYPERACTIVITY (ADHD) DISORDERS EPIDEMICS: DOES FETAL EXPOSURE TO ENDOCRINE DISRUPTING CHEMICALS PLAY A ROLE?}

\author{
M. de Cock, M. Van de Bor \\ Health and Life Sciences, VU University, \\ Amsterdam, The Netherlands
}

Background: The prevalence of neurodevelopmental disorders, such as ASD and $A D H D$, is increasing. This might at least partly be explained by an increased awareness of symptoms and a shift in diagnosis. However, further studies of the incidence of ASD and ADHD are warranted. Evidence has been accumulating that fetal exposure to endocrine disrupting chemicals (EDCs) can cause neurodevelopmental damage.

Objective: To determine a link between fetal EDCs exposure and ASD and/or ADHD.

Methods: A systemic review of the literature (PubMed) was performed. The primary search terms were: 'prenatal exposure delayed effects' and 'EDCs', and 'autistic disorder' or 'attention deficit disorder with hyperactivity'. Secondary search terms were combinations of 'maternal exposure' with 'developmental disabilities/chemically induced' and 'neurotoxins', all with the limiters 'child', 'English', and 'human'.

Results: Fetal exposure to EDCs appears to affect the HPA-axis, leading to disturbed fetal thyroid metabolism and androgen and estrogen homeostasis and therefore possibly disturbed neurodevelopment. Furthermore, fetal exposure to EDCs, organochlorine pesticides in particular, is associated with an elevated risk of ASD and ADHD. Different gene expression in those children due to fetal EDCs exposure is reported.

Conclusion: Evidence is emerging that fetal exposure to EDCs contributes to the increasing prevalence of ASD and ADHD. However, prospective long term cohort studies are necessary to confirm these observations.

\section{NEUROSENSORY IMPAIRMENT AND TREAMENT OF PATENT DUCTUS ARTERIOSUS IN EXTREMELY LOW BIRTH WEIGHT INFANTS}

V.P. Sheriff, M.S. Luna, D.B. Bravo, S. Caballero, M.L. Franco, B. Bernardo, E. Sanz, C. Ramos, E. Zamora, E. Maderuelo, S. Zeballos, M.I. Marsinyach, S. Villar, C.S. Gomez Orgaz, P. Chimenti, G. Zeballos

Pediatrics (Neonatology), Hospital General Universitario Gregorio Marañon, Madrid, Spain

Background and aim: There is controversy in the last years on on surgical closure of the ductus arteriosus in extremely low birth weight infants. Study the possible consequences of surgical closure of the patent ductus arteriosus in the newborn period.

Material and methods: 422 preterm newborns born with of less than $1000 \mathrm{gr}$. were studied, 202 $(47,86 \%)$ had symptomatic PDA (Patent Ductus Arteriosus) and received medical therapy with indomethacine., $36 \quad(8,53 \%)$ underwent PDA ligation. We studied the risks of adverse outcomes ( Retinopathy (ROP), bronchopulmonary dysplasia (BDP), and Neurosensory Impairment (NI)) between both groups, surgical closure and non surgical treatment.

Results: No significant differences between the two groups were found in: Maternal age, gestations, gestacional age, type of delivery, $\mathrm{pH}$ at delivery, Apgar score at 1 and 5 minutes, weight, height and cephalic perimeter. ROP, Intracraneal hemorrage, neumothorax, necrotaizing enterocolitis, had no different incidences in both groups. We found augmented risk in surgery closure group : Lenth of stay (LOS) in hospital $99,5 \pm 6,46$ versus $69,50 \pm 3,59$ $(p<0,001)$, NI Odds ratio $3,149(1,286-7,712)$ and BDP 2,364 (1,085-5, 151).

Conclusions: PDA surgical closure seems that can be associated with increased risk of LOS, $\mathrm{NI}$ and BDP in this group of infants. We could not demonstrate, like other authors have, its relationship with ROP. 